\title{
PULMONARY EMBOLISM AFTER ELBOW ARTHROSCOPY: A CASE REPORT
}

\author{
Young Lae Moon, Sung Woo Sim, Moustafa Elsayed ${ }^{(*)}$ \\ Orthopaedic dept, Chosun University Hospital, South Korea \\ *E-mail: mostafa.ismael79@yahoo.com
}

Received 2/2/2021

Accepted 12/4/2021

\begin{abstract}
Pulmonary embolism after elbow arthroscopy is extremely rare, and no cases have been reported in the literatures. We report the case of a 67-year-old woman who presented by pulmonary embolism after elbow arthroscopy for debridement of medial epicondylitis. Early detection and proper management with Heparin therapy were essential for saving this patient. Physicians must be alert to the possibility of pulmonary embolism after elective elbow arthroscopic procedures.
\end{abstract}

Keyword: Elbow, Arthroscopy, Pulmonary embolism

\section{Introduction}

Pulmonary embolism after elbow arthroscopy is extremely rare. In fact, no such case has been previously reported in the literatures. The authors report a case of a 67 year old woman who presented by pulmonary embolism after elbow arthroscopy for debridement of medial epicondylitis.

\section{Case report}

A 67 year old woman $(163 \mathrm{~cm}$ height, 69 $\mathrm{Kg}$ weight) without known risk factors for deep venous thrombosis (DVT) and not taking any medication, presented with a painful right elbow, which was diagnosed as medial epicondylitis. She underwent arthroscopic debridement. The surgery was performed under general anesthesia, without regional anesthesia, in the lateral decubitus position with the involved side uppermost without traction or a tourniquet. The arm was supported by an assistant with the forearm hanging free and the elbow flexed at 90 degrees. A needle was then inserted at the site of the mid-lateral portal; that is, within the triangle formed by the olecranon, lateral epicondyle, and radial head, and $25 \mathrm{ml}$ of sterile normal saline solution was injected to distend the capsule fully. Proximal anterolateral and proximal anteromedial portals were used during arthroscopy of the elbow. Degeneration at the origin of the common flexor tendon and synovial hypertrophy were observed during surgery. The procedure consisted of simply debridement for medial epicondylitis. The time of surgery was 10 minutes with no complications, and the total anesthesia time was 30 minutes. A long arm splint was used for immobilization during the immediate postoperative period. On the 
first postoperative day, the elbow dressing was changed, and the patient performed normal daily activities. However, on the second day after surgery, she complained of chest pain and shortness of breath. Her vital signs revealed a thread pulse and a blood pressure of 70/50 $\mathrm{mm} \mathrm{Hg}$. She was given nasal oxygen, and her blood pressure was reported to be improved to $90 / 50 \mathrm{~mm} \mathrm{Hg}$. Within 15 minutes, she was transmitted to intensive care unit (ICU). There was no swelling or any other signs of DVT in right upper limb. Chest $\mathrm{X}$-ray demonstrated increased interstitial marking in both lung fields. The diagnosis was confirmed by an angiography scan, which showed bilateral multifocal pulmonary embolism, fig. (1). Heparin therapy was instituted and her condition was improved. A workup for thromboembolic disorders was non-specific. A followup angiography scan performed at 10 days postoperatively showed resolution of the pulmonary embolism, fig. (1). She was discharged home the next day, with an international normalized ratio of 2.5 (normal is 1.0 to 1.5 , but in thrombosis 2.0 to 3.0 is required). After 3 months follow-up she was able to perform daily activities without restrictions.

\section{Discussion}

Although the potential complications of elbow arthroscopy have been previously described, the literatures contain nothing about pulmonary embolism after elbow arthroscopy. Kelly et al [1] conducted a retrospective review of 473 consecutive elbow arthroscopies, and noted serious complications such as, joint infection, prolonged drainage from portal sites, superficial infection, contracture, and temporary nerve palsy. The authors considered that serious or permanent complications were uncommon after elbow arthroscopy. In our case, each portal was established with extreme caution to avoid damage to nearby vessels and nerves. The joint capsule was fully distended, and the elbow was flexed at 90 degrees. In addition, an 18-gauge needle was used to make trial entries into the joint and to help to determine the site for arthroscope introduction. Furthermore, the portals used are known to be optimal in terms of reducing the risk of neurovascular injury [2]. The most common risk factors of pulmonary embolism after upper extremity arthroscopic procedure are; prior DVT, history of pulmonary embolism, coagulation problems, chronic smoking, hypertension, diabetes, prolonged immobilization, obesity, venous stasis and phlebitis, a history of malignancy, and any other condition requiring prolonged bed rest [3]. In a case described by Burkhart [4], underlying Hodgkin's lymphoma was found to induce hypercoagulability. Polzhofer et al [5] reported on a case of upper extremity DVT followed by pulmonary embolus after arthroscopic subacromial decompression in the lateral position, and considered that shaving during subacromial decompression, improper positioning, excessive traction of the arm, or fluid-induced soft-tissue swelling may have irritated and compressed the subclavian vein. Cornwall and Yang [6] reported a case of upper extremity DVT after antegrade femoral nailing. In this case, the arm was positioned across the chest and stabilized with a sheet, and after surgery, the patient complained of extreme right upper extremity pain and swelling. Doppler ultrasonography revealed a large thrombus in the right proximal subclavian vein. The authors considered that positioning of the arm during surgery contributed to the development of the DVT. To test this hypothesis, they placed 
the arm in various positions to determine the effect on venous flow by contrast venography, and found that the cross-body adduction position showed distinct venous compression. This compression was subsequently relieved by placing a bolster on the chest. Kuremsky et al [7] reviewed 1908 arthroscopy files and identified eight cases complicated by symptomatic thromboembolism. There were seven cases of DVT and five cases of pulmonary embolism. Only four of the eight had hemostasis factor abnormalities (protein $\mathrm{S}$ deficiency, associated cancers, and BMI of 35). All these patients had been operated on in the lateral decubitus position. In terms of factors favoring thrombosis, the authors suggested the following: the lateral decubitus position with traction on the limb along its axis, prolonged surgery, use of an interscalene brachial plexus block, and the presence of risk factors for thrombosis (neoplasm, antithrombotic factor deficiency). However, our case differs from previously reported cases, as our patient had no intrinsic risk factors for thromboembolism and no traction was applied to the arm. Furthermore, we did not apply a tourniquet because of the short operation time and no bleeding tendency. Interestingly, in a previous report, tourniquet inflation and deflation were associated with emboli formation $[7,8]$. In our patient, we are unable to determine whether the embolus was the result of arm positioning or other unknown risk factor. This case report warning physicians to be aware that pulmonary embolism can occur after elective elbow arthroscopic procedures.

\section{References}

[1] Araki, S., Uchiyama, M. (1991). Fatal pulmonary embolism following tourniquet inflation. A case report. Acta
Orthop Scand. 62 (5): 488. Kelly, E., Morrey, B., O'Driscoll, S. (2001). Complications of elbow arth-roscopy. J. Bone Joint Surg Am. 83-A (1): 2534.

[2] O'Driscoll, S., Morrey, B. (1992). Arthroscopy of the elbow: Diagnostic and therapeutic benefits and hazards. $\boldsymbol{J}$. Bone Joint Surg Am. 74 (1): 84-94.

[3] Rockwood, Jr C., Wirth, M., Blair S. (2003). Warning: Pulmonary embolism can occur after elective shoulder surgery-report of two cases and survey of the members of the American Shoulder and Elbow Surgeons. J. of Shoulder and Elbow Surgery. 12 (6): 628-630.

[4] Burkhart, S. (1990). Deep venous thrombosis after shoulder arthroscopy. Arthroscopy. 6 (1): 61-63.

[5] Polzhofer, G., Petersen, W., Hassenpflug, J. (2003). Thromboembolic complication after arthroscopic shoulder surgery. Arthroscopy. 19 (9): E129- E132.

[6] Cornwall, R., Yang, E. (2002). Upper extremity deep venous thrombosis following antegrade femoral nailing: case report and venographic study of operative positioning. Orthopedics. 25 (12): 1395-1397.

[7] Kuremsky, M., Lyle Cain, Jr., Fleischli J. (2011). Thromboembolic phenomena after arthroscopic shoulder surgery. Arthroscopy. 27 (12): 1614-1619.

[8] Araki S, Uchiyama M. (1991). Fatal pulmonary embolism following tourniquet inflation. A case report. Acta Orthop Scand. 62 (5):4 88 